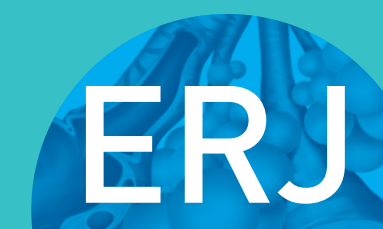

open research
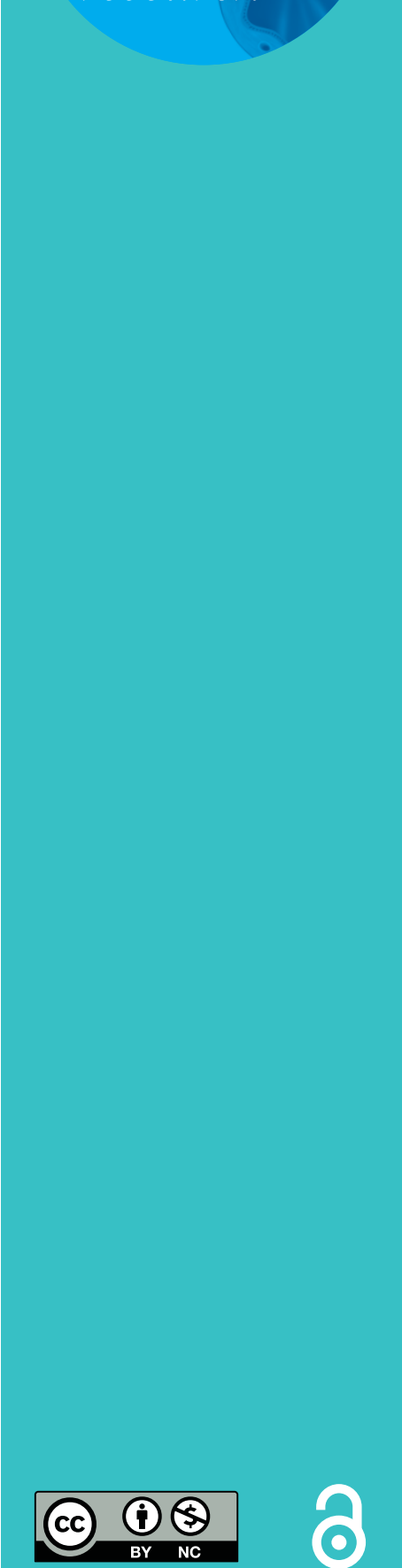

\section{Adaptive servoventilation in clinical practice: beyond SERVE-HF?}

\author{
Winfried Randerath ${ }^{1,5}$, Katja Schumann ${ }^{1,5}$, Marcel Treml ${ }^{1}$, Simon Herkenrath ${ }^{1}$, \\ Alessandra Castrogiovanni ${ }^{1}$, Shahrokh Javaheri ${ }^{2,3}$ and Rami Khayat ${ }^{4}$
}

Affiliations: ${ }^{1}$ Institute of Pneumology at the University of Cologne, Bethanien Hospital, Clinic for Pneumology and Allergology, Centre of Sleep Medicine and Respiratory Care, Solingen, Germany. ${ }^{2}$ Bethesda North Hospital, Cincinnati, OH, USA. ${ }^{3}$ Pulmonary Diseases and Sleep, University of Cincinnati, Cincinnati, OH, USA. ${ }^{4}$ Division of Pulmonary Critical Care and Sleep, Ohio State University, Columbus, OH, USA. ${ }^{5}$ These authors contributed equally.

Correspondence: Winfried Randerath, Institute of Pneumology at the University of Cologne, Bethanien Hospital, Clinic for Pneumology and Allergology, Centre of Sleep Medicine and Respiratory Care, Aufderhöher Str. 169, 42699 Solingen, Germany. E-mail: randerathlaklinik-bethanien.de

ABSTRACT Adaptive servoventilation (ASV) has proven effective at suppressing breathing disturbances during sleep, improving quality of life and cardiac surrogate parameters. Since the publication of the SERVE-HF-trial, ASV became restricted. The purpose of this study was to evaluate the clinical relevance of the SERVE-HF inclusion criteria in real life and estimate the portion of patients with these criteria with or without risk factors who are undergoing ASV treatment.

We performed a retrospective study of all patients who were treated with ASV in a university-affiliated sleep laboratory. We reviewed the history of cardiovascular diseases, echocardiographic measurements of left ventricular ejection fraction (LVEF) and polysomnography.

From 1998 to 2015, 293 patients received ASV, of which 255 (87.0\%) had cardiovascular diseases and $118(40.3 \%)$ had HF. Among those with HF, the LVEF was $\leqslant 45 \%$ in 47 patients (16.0\%). Only 12 patients (4.1\%) had LVEF $<30 \%$. The SERVE-HF inclusion criteria were present in 28 (9.6\%) ASV recipients. Of these patients, 3 died within 30-58 months of therapy, all with systolic HF and a LVEF $<30 \%$.

In this study, only a small minority of ASV patients fell in the risk group. The number of fatalities did not exceed the expected mortality in optimally treated systolic HF patients.

@ERSpublications

The majority of ASV patients do not fulfil the risk criteria. Fatalities under ASV did not exceed expected figures. http://ow.ly/V2HI30fBURh

Cite this article as: Randerath W, Schumann K, Treml M, et al. Adaptive servoventilation in clinical practice: beyond SERVE-HF? ERJ Open Res 2017; 3: 00078-2017 [https://doi.org/10.1183/ 23120541.00078-2017].

Received: June 302017 | Accepted after revision: Aug 272017

Conflict of interest: Disclosures can be found alongside this article at openres.ersjournals.com

Copyright $\odot$ ERS 2017. This article is open access and distributed under the terms of the Creative Commons Attribution Non-Commercial Licence 4.0. 


\section{Introduction}

Sleep disordered breathing (SDB) comprises a variety of pathophysiological and clinical phenotypes [1]. Obstructive sleep apnoea (OSA) is not only characterised by morphological changes of the upper airways, an increase in the critical closing pressure and failing of compensatory muscle function, but also by an increase in the central respiratory drive $[2,3]$.

Central breathing disturbances can be a consequence of various underlying diseases, mainly cardiovascular disorders, but also renal failure, chronic opioid use, or neurological diseases. Besides the classical subtypes (central sleep apnoea (CSA) and periodic breathing), many patients present with coexisting OSA and central SDB in different combinations. Non-hypercapnic or hypocapnic central breathing disturbances are associated with a shift between hyperventilation and hypoventilation, increased chemosensitivity and brainstem [4-6]. This results in instability of respiration, clinically characterised by a periodic breathing pattern, as in heart failure (HF), high altitude, or treatment-emerging sleep apnoea [1].

Continuous positive airway pressure (CPAP) is the first therapeutic approach for most patients with obstruction of the upper airways and non-hypercapnic CSA [1]. However, it fails in many patients with pure or coexisting central SDB, especially in those with a high loop gain of ventilatory regulation [7]. Adaptive servoventilation (ASV) has been developed to address these complicated situations. It applies positive expiratory pressure to overcome upper airway obstruction, pressure support during inspiration to counterbalance periods of hypoventilation, and mandatory breaths to override central apnoeas $[8,9]$. ASV has been used in various entities of central SDB and coexisting obstructive and central sleep apnoea [10-13]. It has proven superior to other treatment options in normalising respiration, improving sleep quality and quality of life, and left ventricular ejection fraction (LVEF) in chronic systolic HF patients [14-16].

CowIE et al. [17] studied the effect of ASV on survival in patients with severe systolic HF (HF with reduced ejection fraction: HFrEF) and predominant CSA. Although the study did not show a significant difference between ASV and conventional cardiac therapy in the primary composite cardiac outcome parameter, an exploratory analysis found that ASV was associated with increased death from any cause and death from cardiovascular diseases. Serious concerns were raised regarding the methodology, design and interpretation of the data [18]. Moreover, our experience supports a survival benefit in patients with HFrEF who pursued ASV treatment initiated after hospitalisation for acute cardiac decompensation [19] (KHAYAT et al., unpublished observations). However, ASV device manufacturers and some health authorities recommend against the use of ASV in patients who fulfil the SERVE-HF inclusion criteria (i.e. predominant CSA and chronic systolic HF with substantially reduced LVEF $\leqslant 45 \%)$. This recommendation may be difficult to translate in clinical practice for several reasons.

- The predominance of one or another phenotype of SDB is often hard to determine clinically. This particularly applies to hypopnoeas, which are difficult to differentiate accurately (central versus obstructive), but represent the majority of the apnoea-hypopnea index (AHI) in most patients. In addition, the precise discrimination of obstructive and central events may require full polysomnography [20].

- Different authors use different percentages of the central AHI/total AHI ratio to define CSA versus OSA.

- Baseline echocardiograms for evaluation of the LVEF are not always available.

These practical problems may lead clinicians to unnecessarily withhold ASV from patients without actual risk factors. Therefore, the purpose of this study was to evaluate the clinical relevance of the SERVE-HF inclusion criteria to the practice of sleep medicine in a real-life population and to estimate the portion of patients with or without risk under treatment with ASV.

\section{Material and methods}

Patients and design

We reviewed our sleep centre's database to identify all the patients who received ASV between 1998 and 2015. These patients were contacted for participation in the study. Patients then provided demographic, sleep and functional data. In addition, full demographic, objective sleep, cardiovascular and vital data were obtained from medical records. In some cases, outside practitioners or institutions were contacted to obtain required data. We then determined a cohort of ASV recipients who fulfilled the inclusion criteria for SERVE-HF.

A stepwise algorithm was used in identifying the cohort (detailed in figure 1) including the following aspects.

1. Initiation of treatment with ASV

2. Presence or absence of cardiovascular diseases based on patients' history and information from general practitioners or cardiologists

3. Presence or absence of chronic heart failure

4. Presence or absence of LVEF $\leqslant 45 \%$ 


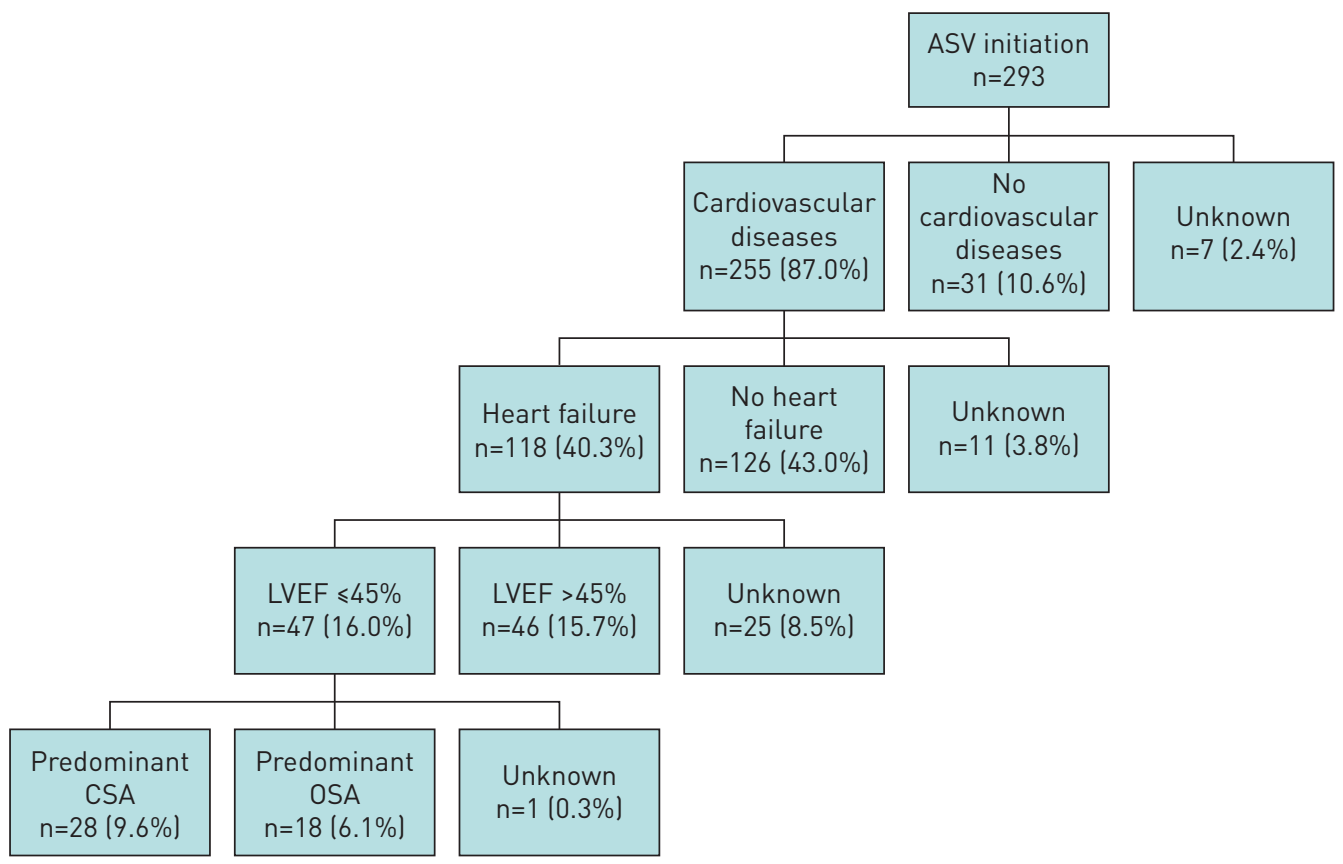

FIGURE 1 Algorithm to define different patient groups. Percentages are of all patients with adaptive servoventilation (ASV) initiation. LVEF: left ventricular ejection fraction; CSA: central sleep apnoea; OSA: obstructive sleep apnoea.

5. Type of SDB and the predominance of central breathing disturbances

\section{Devices}

Nine ASV devices from three different manufacturers were used (SomnoVent CR, SomnoVent Auto ST, from Weinmann, Germany; BiPAP autoSV, BiPAP autoSV 2, BiPAP autoSV 2 (with auto-EPAP), BiPAP autoSV Advanced SO, from Philips Respironics, USA; AutoSet CS, AutoSet CS 2, AutoSet CS PaceWave, from ResMed, USA). Every manufacturer provided different generations of development devices with automatic expiratory positive airway pressure (auto-EPAP) and/or a pressure support that can be reduced to zero (Group II) and devices without any of these features (Group I) (table 1).

\section{Polysomnography}

All patients underwent in-hospital supervised polysomnography (PSG) for diagnosis and treatment initiation using SOMNOlab (Weinmann, Hamburg, Germany) or Alice 4 or 5 (Philips Respironics, Murrysville, PA, USA) as previously described [11, 20]. An apnoea was defined as the cessation of respiratory flow for $\geqslant 10 \mathrm{~s}$. Central apnoeas were scored if respiratory effort was absent. A hypopnoea was defined as a reduction in nasal pressure signal (flow) of $\geqslant 30 \%$ for $\geqslant 10 \mathrm{~s}$ accompanied by an arousal and/ or a decrease in oxygen saturation of $\geqslant 3 \%$. Hypopnoeas were differentiated based on flattening of airflow, paradoxical breathing, pattern of ventilation, position of arousal relative to SDB and sleep stage, as described previously [20].

\section{Echocardiography}

In-house echocardiograms were performed by certified cardiologists (Vivid S 6, GE Healthcare, USA). The LVEF was calculated using Simpson's and/or Teichholz's methods. For all in-house echocardiograms, LVEF was quantified. For a few patients in whom echocardiography was performed externally, we only had a qualitative description of the left ventricular functional status. In this case, we determined the mean value of the functional left ventricular status by using the internationally accepted classification from LANG et al. [21]. Here, a normal LVEF is defined as $\geqslant 55 \%$, a slight reduction as $45-54 \%$, a moderate reduction $30-44 \%$ and a severe reduction is $<30 \%$.

\section{Statistical analysis}

Data are given as absolute numbers, proportions or mean (standard deviation), unless stated otherwise. Statistical calculations were carried out with IBM SPSS Statistics (IBM SPSS Statistics for Windows, Version 24.0, IBM, Armonk, NY). Comparisons of more than two groups were done by one-way ANOVA, and Tukey's post hoc tests, paired or unpaired t-tests were used to compare two groups, as appropriate. 
TABLE 1 Anthropometric data

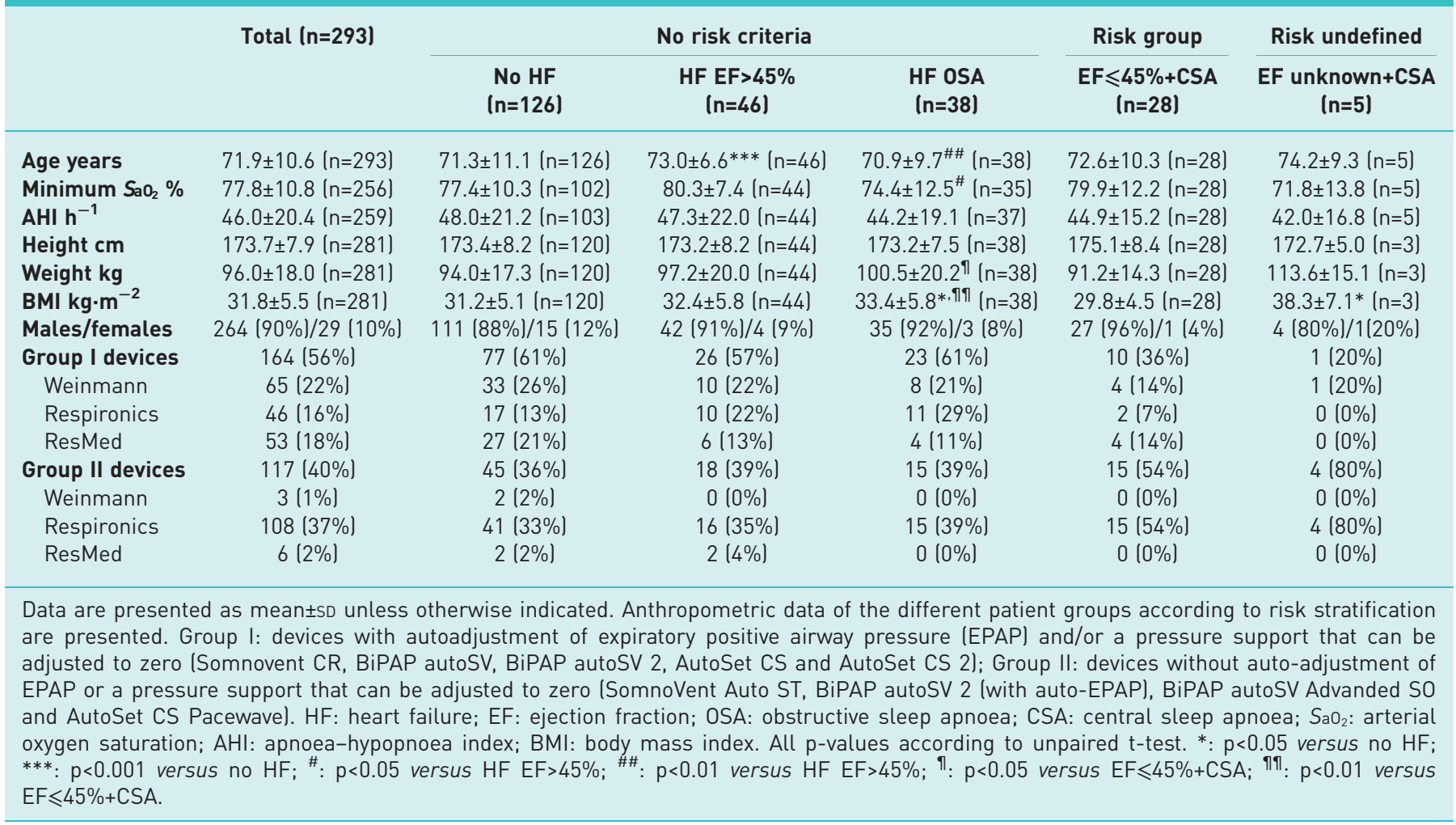

\section{Results}

During the study period, ASV treatment was initiated in 293 patients. The mean age was 71.9 years (SD 10.6), the AHI was $46.4 / \mathrm{h}(20.5 / \mathrm{h})$, the lowest oxygen saturation $\left(\mathrm{SaO}_{2} \mathrm{~min}\right)$ was $78 \%(10.8 \%)$, and the body mass index $31.8 \mathrm{~kg} \cdot \mathrm{m}^{-2}\left(5.5 \mathrm{~kg} \cdot \mathrm{m}^{-2}\right)$. Out of the patients, $264(90 \%)$ were male and 29 were female (10\%) (table 1). The sleep medical diagnoses were CSA (57\%), including periodic breathing (36\%); OSA (26\%); treatment-associated CSA (8\%); coexisting obstructive and central sleep apnoea (4\%); obesity hypoventilation syndrome (1\%); and others (4\%).

Of the 293 ASV patients, 255 (87.0\%) suffered from at least one cardiovascular comorbidity, including 227 of $255(89 \%)$ with arterial hypertension (table 2), and $118(46 \%)$ of 255 of the patients suffered from systolic or diastolic HF as a consequence of, or in association with, arterial hypertension (87\%), coronary artery disease (60\%), arrhythmias (47\%), aortic valve disease (25\%) and dilated cardiomyopathy (15\%).

\section{Characteristics of the HF patients in the dataset}

LVEF measurements were available in $79 \%$ of HF patients ( $n=118)$. HFrEF $\leqslant 45 \%$ was present in 47 of the HF patients (39.8\% of the HF patients; $16.0 \%$ of the whole ASV population) (table 3). Predominant CSA

TABLE 2 Cardiovascular diseases in 255 patients with at least one cardiovascular comorbidity

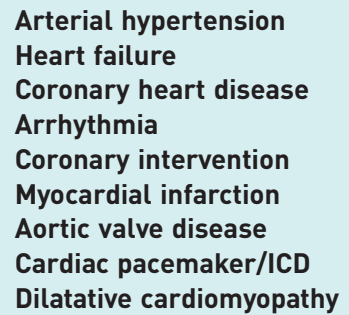

ICD: implantable cardiac defibrillator. 
TABLE 3 Left ventricular ejection fraction in the adaptive servoventilation population with heart failure

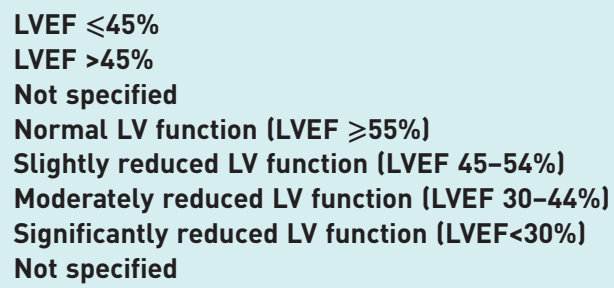

LVEF: left ventricular ejection fraction; LV: left ventricular.

(more than $50 \%$ central AHI) was present in 28 patients with $\mathrm{HFrEF} \leqslant 45 \%$, while 18 patients had predominant OSA, and the type of SDB could not be determined in one patient (table 4).

The Epworth sleepiness scale (ESS) decreased from 7.8 (4.5) at baseline to $5.4(3.7)$ with treatment $(\mathrm{p}<0.001$, pairwise availability of data in 75 cases, time frame $27 \pm 26$ months) in the HF population. Thirty of 94 patients $(32 \%)$ had an ESS score $\geqslant 10$ pre-treatment, compared to only 15 patients out of 91 (16\%) with treatment. Information allowing for assessment of CSA predominance was available in 46 of those cases.

Of all the patients with HFrEF $\leqslant 45 \%, 28$ (9.6\%) presented with predominant CSA; 25 of these showed periodic breathing. The LVEF was between $30 \%$ and $45 \%$ in 20 (6.8\% of whole population) patients, while it was $<30 \%$ in $8(2.7 \%)$. Patients with LVEF $\leqslant 45 \%$ and predominant OSA showed a significantly higher index of respiration-related arousals than those with predominant CSA.

All patients who fulfilled the inclusion criteria of HFrEF predominant CSA and those with unclear information were contacted directly or via the general practitioner. There were only 3 deaths in the 28 patients who fulfilled the inclusion criteria of SERVE-HF. These 3 patients had LVEF $<30 \%$.

TABLE 4 Data from diagnostic polysomnography in patients with left ventricular ejection fraction $\leqslant 45 \%$

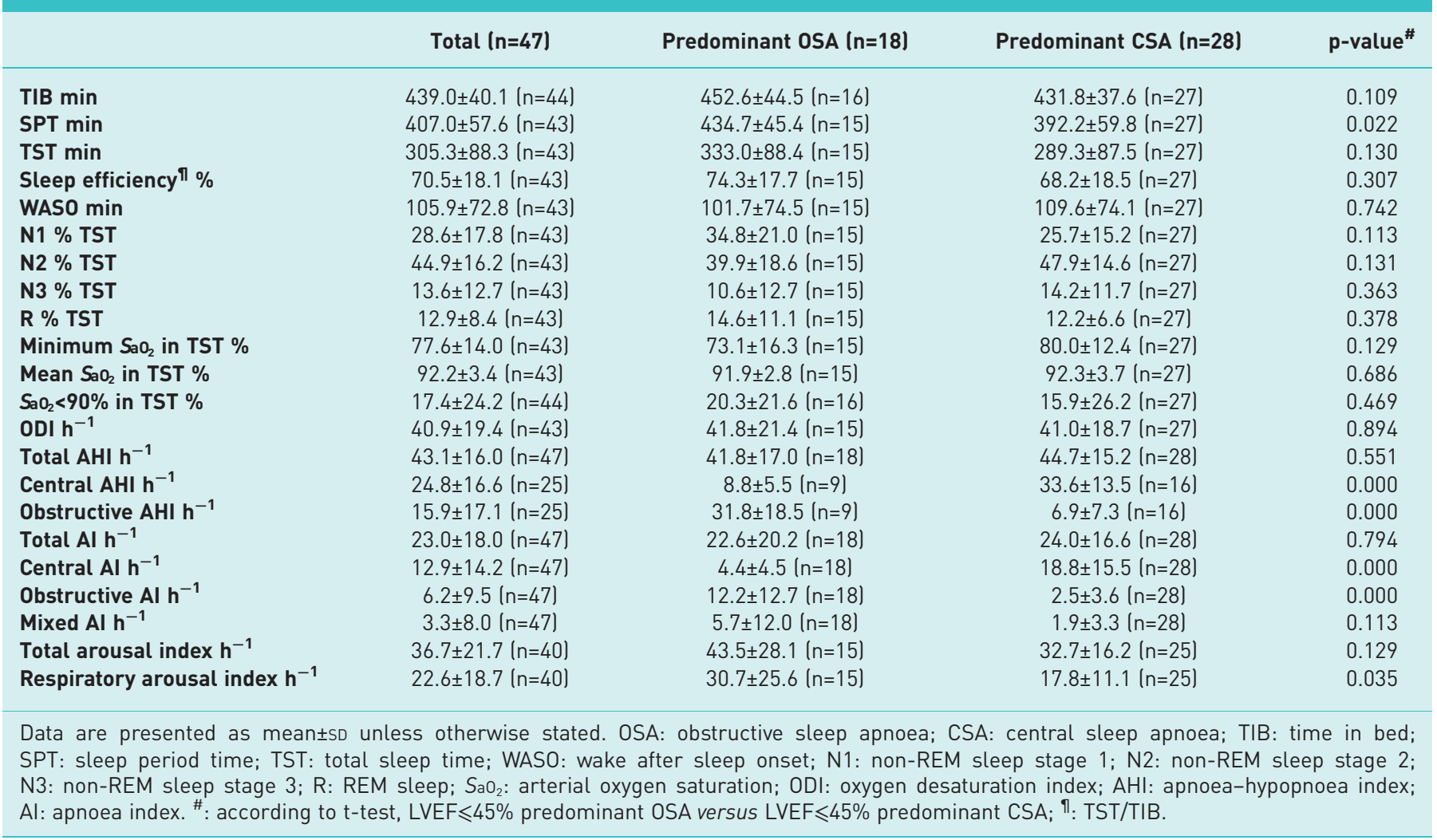


Six patients had stopped treatment, 3 had changed therapy to CPAP, APAP or bilevel. The dates of deaths were available in 2 of 3 patients and happened after 30 and 58 months of ASV therapy.

\section{Discussion}

This analysis of a large unselected group of patients treated with ASV showed that only a minority of patients (9.6\%) fulfilled the risk criteria as described in the SERVE-HF trial. The majority of patients either did not meet the high-risk criteria or were treated with ASV for indications other than predominant CSA and no change in therapy would have been needed. In this population, ASV was initiated when patients presented with pure CSA or in combination with obstructive disturbances if a treatment with CPAP had failed. Thus, the initiation of ASV was not limited to HF patients.

The SERVE-HF trial investigated the impact of ASV as compared to optimal cardiac care on mortality and serious cardiac events in patients with $\mathrm{HFrEF} \leqslant 45 \%$ and predominant CSA. Although the study failed to show a difference in the primary combined outcome parameter, the observed excess all-cause mortality and mortality due to cardiovascular events in the ASV group led to safety warnings from healthcare authorities and manufacturers. The SERVE-HF inclusion criteria were generalised as contraindications for the use of ASV. Although these exclusion criteria were precisely defined, clinicians became concerned regarding the use of ASV in general. The purpose of this study was 1) to identify individual patients at risk in order to find clinical solutions for them, and 2) to evaluate the clinical relevance of SERVE-HF in a real-life population.

Although a majority of the patients treated with ASV in our study had at least one cardiovascular disease (87.0\%), only $40.3 \%$ presented with HF and only $16.0 \%$ with $\mathrm{HFrEF} \leqslant 45 \%$. More recent analyses of the SERVE-HF data confirmed that the mortality risk is limited to patients with even more severely impaired LVEF. In an adjusted analysis, EulEnburg et al. [22] showed that the risk of cardiovascular deaths without previous hospital admission was limited to those with an ejection fraction $<30 \%$. Taking these aspects into consideration, the number of patients at risk in our group would be reduced to $2.7 \%$. All three fatalities occurred in the group of patients with LVEF $<30 \%$. Available data from these patients showed a treatment period of 30 to 58 months. Based on large, prospective studies, the mortality rate in these patients would be estimated as $25 \%$ after 2.5 years, $35-40 \%$ after 4 years and $55-60 \%$ after 5 years $[23,24]$. Thus, there is no indication of an increased number of unexpected deaths. Although this information has to be approached with caution because of the small number of events, it confirms the EULENBURG data limiting the risk - if any - to the patients with most severely reduced LVEF.

The discrimination of obstructive and central disturbances is of crucial importance. Most studies on ASV focus on patients with pure CSA/periodic breathing. However, many patients suffer from coexistence of OSA and CSA with different relative proportions of the phenotypes. In patients with marginal differences between obstructive and central disturbances, in particular, a precise differentiation of the hypopnoeas is of crucial relevance, because they often represent the vast majority of breathing disturbances. For this analysis, we followed the algorithm of hypopnoea differentiation as previously described [20], showing that there was a predominance of CSA in only just over half of the HFrEF patients (28 out of 47).

Our study is limited by its retrospective design. Moreover, information on cardiovascular comorbidities is based on information provided by the patients and general practitioners and our own data files. However, according to the authoritative obligations and the need for optimal patient care, we re-evaluated the patients whenever possible in unclear situations. However, our findings are in line with data from another group [25].

In conclusion, the overwhelming majority of ASV patients are treated for central sleep apnoea and coexisting obstructive and central sleep apnoea without being at risk according to the SERVE-HF trial. Fatalities were limited to the most severe HF patients and did not exceed the estimated numbers.

\section{References}

1 Randerath W, Verbraecken J, Andreas S, et al. Definition, discrimination, diagnosis, and treatment of central breathing disturbances during sleep. An ERS statement. Eur Respir J 2017; 49: 1600959.

2 Wellman A, Eckert DJ, Jordan AS, et al. A method for measuring and modeling the physiological traits causing obstructive sleep apnea. J Appl Physiol (1985) 2011; 110: 1627-1637.

3 Javaheri S, Barbe F, Campos-Rodriguez F, et al. Sleep apnea: types, mechanisms, and clinical cardiovascular consequences. J Am Coll Cardiol 2017; 69: 841-858.

4 Solin P, Roebuck T, Johns DP, et al. Peripheral and central ventilatory responses in central sleep apnea with and without congestive heart failure. Am J Respir Crit Care Med 2000; 162: 2194-2200.

5 Lorenzi-Filho G, Azevedo ER, Parker JD, et al. Relationship of carbon dioxide tension in arterial blood to pulmonary wedge pressure in heart failure. Eur Respir J 2002; 19: 37-40.

6 Javaheri S. A mechanism of central sleep apnea in patients with heart failure. N Engl J Med 1999; 341: 949-954.

7 Sands SA, Edwards BA, Kee K, et al. Loop gain as a means to predict a positive airway pressure suppression of Cheyne-Stokes respiration in patients with heart failure. Am J Respir Crit Care Med 2011; 184: 1067-1075. 

operational algorithms). Chest 2014; 146: 514-523.

9 Javaheri S, Brown LK, Randerath WJ. Clinical applications of adaptive servoventilation devices: part 2. Chest 2014; 146: $858-868$.

10 Javaheri S, Harris N, Howard J, et al. Adaptive servoventilation for treatment of opioid-associated central sleep apnea. J Clin Sleep Med 2014; 10: 637-643.

11 Randerath WJ, Nothofer G, Priegnitz C, et al. Long-term auto servo-ventilation or constant positive pressure in heart failure and co-existing central with obstructive sleep apnea. Chest 2012; 142: 440-447.

12 Ramar K, Ramar P, Morgenthaler TI. Adaptive servoventilation in patients with central or complex sleep apnea related to chronic opioid use and congestive heart failure. J Clin Sleep Med 2012; 8: 569-576.

13 Dellweg D, Kerl J, Hoehn E, et al. Randomized controlled trial of noninvasive positive pressure ventilation (NPPV) versus servoventilation in patients with CPAP-induced central sleep apnea (complex sleep apnea). Sleep 2013; 36: 1163-1171.

14 Sharma BK, Bakker JP, McSharry DG, et al. Adaptive servoventilation for treatment of sleep-disordered breathing in heart failure: a systematic review and meta-analysis. Chest 2012; 142: 1211-1221.

15 Pepperell JC, Maskell NA, Jones DR, et al. A randomized controlled trial of adaptive ventilation for Cheyne-Stokes breathing in heart failure. Am J Respir Crit Care Med 2003; 168: 1109-1114.

16 Hetzenecker A, Roth T, Birner C, et al. Adaptive servo-ventilation therapy of central sleep apnoea and its effect on sleep quality. Clin Res Cardiol 2016; 105: 189-195.

17 Cowie MR, Woehrle H, Wegscheider K, et al. Adaptive servo-ventilation for central sleep apnea in systolic heart failure. $N$ Engl J Med 2015; 373: 1095-1105.

18 Javaheri S, Brown LK, Randerath W, et al. SERVE-HF: more questions than answers. Chest 2016; 149: 900-904.

19 Khayat R, Jarjoura D, Porter K, et al. Sleep disordered breathing and post-discharge mortality in patients with acute heart failure. Eur Heart J 2015; 36: 1463-1469.

20 Randerath WJ, Treml M, Priegnitz C, et al. Evaluation of a noninvasive algorithm for differentiation of obstructive and central hypopneas. Sleep 2013; 36: 363-368.

21 Lang RM, Bierig M, Devereux RB, et al. Recommendations for chamber quantification: a report from the American Society of Echocardiography's Guidelines and Standards Committee and the Chamber Quantification Writing Group, developed in conjunction with the European Association of Echocardiography, a branch of the European Society of Cardiology. J Am Soc Echocardiogr 2005; 18: 1440-1463.

22 Eulenburg C, Wegscheider K, Woehrle H, et al. Mechanisms underlying increased mortality risk in patients with heart failure and reduced ejection fraction randomly assigned to adaptive servoventilation in the SERVE-HF study: results of a secondary multistate modelling analysis. Lancet Respir Med 2016; 4: 873-881.

23 Moss AJ, Zareba W, Hall WJ, et al. Prophylactic implantation of a defibrillator in patients with myocardial infarction and reduced ejection fraction. N Engl J Med 2002; 346: 877-883.

24 Bardy GH, Lee KL, Mark DB, et al. Amiodarone or an implantable cardioverter-defibrillator for congestive heart failure. N Engl J Med 2005; 352: 225-237.

25 Brill AK, Pichler Hefti J, Geiser T, et al. The SERVE-HF safety notice in clinical practice - experiences of a tertiary sleep center. Sleep Med 2017; 37: 201-207. 